Original

\title{
Comparación de los índices PROFUND y PALIAR en pacientes pluripatológicos con enfermedad crónica no oncológica en fase avanzada
}

\author{
Jesús Díez-Manglano a,b,*, Noelia Gómez-Aguirre ${ }^{c}$, José Velilla-Marco d ${ }^{\mathrm{d}}$, María Pilar Lambán Aranda ${ }^{\mathrm{a}}$, \\ Begoña de Escalante Yangüela ${ }^{e}$, Daniel Fuertes Ruiz ${ }^{a}$, Alfonso García Aranda ${ }^{d}$, \\ Carolina Clemente Sarasa ${ }^{a}$ y en nombre de los investigadores del estudio PALIARAGON \\ a Servicio de Medicina Interna, Hospital Royo Villanova, Zaragoza, España \\ b Grupo de Investigación en Comorbilidad y Puripatología de Aragón, Instituto Aragonés de Ciencias de la Salud, Zaragoza, España \\ c Servicio de Medicina Interna, Hospital Ernest Lluch, Calatayud, España \\ d Servicio de Medicina Interna, Hospital Universitario Miguel Servet, Zaragoza, España \\ e Servicio de Medicina Interna, Hospital Clínico Universitario Lozano Blesa, Zaragoza, España
}

\section{INFORMACIÓN DEL ARTÍCULO}

\section{Historia del artículo:}

Recibido el 22 de agosto de 2018

Aceptado el 31 de enero de 2019

On-line el xxx

\section{Palabras clave:}

Paciente pluripatológico

Enfermedad crónica avanzada

Mortalidad

Poder de discriminación

Estudio de cohortes

\begin{abstract}
R E S U M E N
Fundamento y objetivo: Comparar la rentabilidad de los índices PALIAR y PROFUND para predecir la mortalidad en pacientes pluripatológicos con enfermedad crónica no oncológica en fase avanzada. Material y métodos: Estudio de cohortes, prospectivo y multicéntrico con pacientes pluripatológicos con enfermedades crónicas no oncológicas en fase avanzada ingresados en departamentos de medicina interna entre el 1 de julio y el 31 de diciembre de 2014. Se recogieron datos de edad, género, categorías de pluripatología, enfermedad avanzada, comorbilidad, valoración funcional y cognitiva, síntomas de enfermedad terminal, necesidad de cuidador, ingresos en los 3 y 12 meses previos, número de fármacos, y se calcularon los índices PROFUND y PALIAR. Tras un seguimiento durante 12 meses la mortalidad se valoró con las curvas de supervivencia de Kaplan-Meier y la rentabilidad de los índices con las curvas ROC.

Resultados: Se incluyeron 213 pacientes con edad media 83 (7) años y 106 (49,8\%) eran mujeres. La mortalidad a los 6 meses fue del $40,4 \%$ y a los 12 del 50,2\%. Los pacientes fallecidos puntuaban más alto en los índices PROFUND [11,2(4,2) frente a 8,5(3,9); $\mathrm{p}<0,001]$ y PALIAR [6,7 $(4,6)$ frente a 3,6 $(3,1)$; $\mathrm{p}<0,001$ ]. La capacidad discriminativa del índice PALIAR a los 6 meses (área bajo la curva 0,734; IC95\% $0,665-0,803$ ) fue superior a la del índice PROFUND y no hubo diferencias a los 12 meses.

Conclusiones: En pacientes pluripatológicos con enfermedad crónica en fase avanzada el índice PALIAR tiene un rendimiento mayor que el índice PROFUND para predecir la mortalidad a los 6 meses y similar a los 12 meses.

(C) 2019 Elsevier España, S.L.U. Todos los derechos reservados.
\end{abstract}

\section{Comparison between PROFUND and PALIAR indexes in polypathological patients with advanced non-oncologic chronic diseases}

\section{A B S T R A C T}

Background and objective: To compare the discrimination power of PROFUND and PALIAR indexes for predicting mortality in polypathological patients with advanced non-oncologic chronic disease.

Material and methods: Prospective multicentre cohort study. We included polypathological patients with advanced non-oncologic chronic disease, who were admitted to internal medicine departments between July 1 st and December 31th, 2014. Data was collected from each patient on age, sex, categories of
Keywords: Advanced chronic disease Mortality

Discrimination power

Cohort study

\footnotetext{
* Autor para correspondencia.

Correo electrónico: jdiez@aragon.es (J. Díez-Manglano).

La lista de investigadores del estudio PALIARAGON está disponible en el anexo
} 
polypathology, advanced disease, comorbidity, functional and cognitive assessment, terminal illness symptoms, need for caregiver, hospitalisation in the past three and 12 months and number of drugs. We calculated the PROFUND and PALIAR indexes and conducted a 12-month follow-up. We assessed mortality with the Kaplan-Meier survival curves and the discrimination of indexes with the ROC curves. Results: We included 213 patients with a mean (standard deviation) age of 83.0 (7.0) years, 106 (49.8\%) of whom were female. Mortality at six months was $40.4 \%$ and at 12 months $50.2 \%$. Deceased patients scored higher scores on the PROFUND [11.2(4.2) vs 8.5(3.9); $P<.001$ ] and PALIAR [6.7 (4.6) vs 3.6(3.1); $\mathrm{p}<0,001$ ] indexes. The discrimination of PALIAR index at six months (under the curve area $0.73495 \% \mathrm{CI} 0.665-0.803$ ) was higher than of PROFUND, and there was no difference at 12 months.

Conclusions: In polypathological patients with advanced non-oncologic chronic disease, the PALIAR index had better discrimination power than PROFUND index at 66 months and there were no differences at 12 months.

(C) 2019 Elsevier España, S.L.U. All rights reserved.

\section{Introducción}

Con el envejecimiento de la población y el aumento de las enfermedades crónicas, la mutimorbilidad y la pluripatología son situaciones clínicas cada vez más frecuentes. En ocasiones, a lo largo de su evolución, las enfermedades crónicas progresan hasta provocar insuficiencias de los órganos vitales que llevan a la muerte.

Los pacientes con pluripatología tienen varias enfermedades de carácter progresivo que provocan discapacidad y que con frecuencia están relacionadas entre sí $^{1}$. Sin embargo, en ocasiones una, o a veces más de una, de estas enfermedades progresa a fases avanzadas. La mortalidad en los pacientes pluripatológicos (PP) y en los pacientes con enfermedades en fase avanzada es alta. Por ello establecer un pronóstico puede ayudar, tanto a los pacientes y familiares como a los sanitarios, a organizar planes de cuidados apropiados a la situación de cada individuo.

En los últimos años se han desarrollado en España 2 índices para establecer el pronóstico en pacientes crónicos, los índices PROFUND y PALIAR ${ }^{2,3}$. Son índices multidimensionales que incluyen variables demográficas, clínicas, funcionales y analíticas. El índice PROFUND ayuda a establecer el pronóstico al año en PP y el índice PALIAR a los 6 meses en pacientes con enfermedades crónicas no oncológicas en fase avanzada (ECNOFA). Ambos índices han sido validados externamente $^{4,5}$. Sin embargo, no se ha estudiado cuál de estos índices es más adecuado en los PP que también presentan ECNOFA.

El objetivo de nuestro estudio fue comparar la rentabilidad de los índices PALIAR y PROFUND para predecir la mortalidad a los 6 y 12 meses en PP con ECNOFA.

\section{Métodos}

El estudio PALIARAGON fue un estudio observacional, de cohortes, prospectivo y multicéntrico en el que participaron 5 hospitales de Aragón, España (anexo 1), que ha sido descrito previamente ${ }^{5}$.

\section{Criterios de inclusión y exclusión}

En el período comprendido entre el 1-07-2014 y el 31-12-2014 se incluyeron de forma consecutiva todos los pacientes con una o varias ECNOFA atendidos por los médicos investigadores Los criterios de inclusión y exclusión se presentan como material suplementario en la web (tablas S1 y S2). Solo se consideró el primer ingreso de cada paciente, excluyendo los reingresos posteriores. Para este análisis hemos incluido a los pacientes que cumplían los criterios de PP establecido por la Junta de Andalucía ${ }^{1}$.

\section{Mediciones}

En el momento de ingreso en el hospital se administró a los pacientes un cuestionario exhaustivo con datos demográficos y clínicos relevantes. Los datos recogidos incluyeron la edad, sexo, vivir en el domicilio particular o en una residencia, vivir solo o acompañado, cuidador principal, ECNOFA, comorbilidad, categorías y criterios de pluripatología, capacidad previa al ingreso para realizar las actividades básicas de la vida diaria, función cognitiva, síntomas de enfermedad terminal, situación funcional, número de fármacos consumidos de forma crónica, valores de albúmina y hemoglobina, número de ingresos hospitalarios en los 3 y 12 meses previos, necesidad y disponibilidad de un cuidador, y los índices PROFUND y PALIAR ${ }^{2,3}$. La comorbilidad se midió con el índice de Charlson, que incluye 19 enfermedades ${ }^{6}$. La capacidad para realizar las actividades básicas de la vida diaria se midió con el índice de Barthel que evalúa 10 actividades: comer, bañarse, vestirse, arreglarse, micción, defecación, ir al retrete, trasladarse, deambular y subir escaleras ${ }^{7}$. Puntúa de 0 a 100 y cuanto menor es la puntuación mayor es la discapacidad. Se consideró que un paciente era dependiente cuando la puntuación fue menor de 60 . La función cognitiva se midió con el Short Portable Mental Status Questionnaire (SPMSQ) que consta de 10 preguntas dicotómicas y se considera que hay deterioro cognitivo cuando se producen más de 3 respuestas incorrectas ${ }^{8}$. Se consideró que un paciente necesitaba cuidador cuando el índice de Barthel era menor de 60 o la puntuación en el SPMSQ era superior a 3 errores. La situación funcional se midió con el Eastern Cooperative Oncology Group Performance Status (ECOG-PS $)^{9}$ El insomnio, el dolor crónico, las úlceras por presión, la anorexia, la astenia, las náuseas o vómitos, la diarrea, los edemas, la disnea de reposo y el delirium se consideraron síntomas de enfermedad terminal. La enfermedad terminal se definió con los criterios de la National Hospice Organization ${ }^{10}$.

El índice PROFUND es un índice para valorar la probabilidad de muerte al cabo de un año en PP e incluye también dimensiones demográficas, clínicas, analíticas y funcionales ${ }^{2}$. Recoge las variables edad $\geq 85$ años ( 3 puntos), neoplasia activa (6 puntos), demencia (3 puntos), disnea clase III-IV de la New York Heart Association o 3-4 de la Medical Research Council (3 puntos), delirium en el último ingreso ( 3 puntos), hemoglobina $<10 \mathrm{~g} / \mathrm{dl}$ ( 3 puntos), índice de Barthel $<60$ (4 puntos), ausencia de cuidador o cuidador diferente al cónyuge ( 2 puntos) y 4 o más ingresos hospitalarios en los últimos 12 meses ( 3 puntos). Puntúa entre 0 y 30 puntos y establece 4 niveles de riesgo de muerte: bajo ( $0-2$ puntos), intermedio (3-6 puntos), alto (7-10 puntos) y muy alto (11-30 puntos).

El índice PALIAR es un índice para valorar la probabilidad de muerte al cabo 6 meses en pacientes con ECNOFA e incluye dimensiones demográficas, clínicas, analíticas, y funcionales ${ }^{3}$. Recoge las siguientes variables: edad $\geq 85$ años ( 3 puntos), disnea clase 
funcional iv de la New York Heart Association o disnea 4 de la escala de la Medical Research Council (3,5 puntos), anorexia (3,5 puntos), úlceras por presión ( 3 puntos), albúmina $<2,5 \mathrm{~g} / \mathrm{dl}$ ( 4 puntos) y puntuación ECOG-PS $\geq 3$ ( 4 puntos). Puntúa entre 0 y 21 puntos y establece 4 niveles de riesgo de muerte: bajo ( 0 puntos), intermedio (3-3,5 puntos), alto (4-7 puntos) y muy alto (7,5-21 puntos).

Se realizó un seguimiento de los pacientes durante 12 meses para conocer la supervivencia. El seguimiento se realizó en consulta externa, por revisión de la historia clínica, por llamada telefónica al paciente $o$ a sus familiares o cuidadores, o consultando el Índice Nacional de Defunciones ${ }^{11}$. Se siguió a los pacientes de forma ciega, es decir, los investigadores no sabían el valor del índice PALIAR o PROFUND del paciente cuando evaluaron el resultado.

El estudio fue aprobado por el Comité Ético de Investigación Clínica de Aragón (PI 14/0056). Todos los pacientes (o sus cuidadores en caso de deterioro cognitivo) firmaron un consentimiento informado escrito.

\section{Análisis estadístico}

Las variables cuantitativas se expresaron como media (desviación estándar). Las comparaciones entre grupos se realizaron con el test de la t de Student. Las variables cualitativas se expresaron como número absoluto y porcentaje. La comparación de variables cualitativas se realizó con el test de chi-cuadrado, utilizando la corrección de Yates y en caso preciso con el test exacto de Fisher.

La relación entre los índices se valoró con el test de correlación de Pearson. La capacidad discriminativa de los instrumentos pronósticos se comprobó estimando las curvas de supervivencia de Kaplan-Meier para los grupos de riesgo según las puntuaciones de los índices PALIAR y PROFUND. El poder de discriminación de ambos índices se valoró calculando el área bajo la curva ROC y el estadístico $C$ a los 6 y 12 meses.

Para todos los casos se estableció el nivel de significación estadística para un valor de $\mathrm{p}<0,05$.

El análisis de los datos se realizó con el programa estadístico IBM Corp. Released 2013 IBM SPSS Statistic for Windows, Versión 22.0, Armonk, NY, EE. UU.

\section{Resultados}

\section{Características de los pacientes}

Se incluyeron 213 pacientes, con una edad media de 83 (7) años y 106 (49,8\%) fueron mujeres. Las características de los pacientes se presentan en la tabla 1 . Nuestros pacientes tenían una capacidad limitada para las actividades básicas de la vida diaria medida con el índice de Barthel y deterioro cognitivo según el SPMSQ. Por esta razón un $67,1 \%$ precisaban tener un cuidador.

En nuestro estudio los pacientes tenían con mayor frecuencia enfermedades cardíacas (62\%) aunque las enfermedades en fase avanzada más frecuentes eran de origen neurológico (52,1\%). Padecían más de una ECNOFA 49 (23\%) pacientes. Además 90 pacientes $(40,2 \%)$ cumplían criterios de enfermedad terminal de la National Hospice Organization. Los síntomas de enfermedad terminal más frecuentes fueron los edemas, el estreñimiento, la anorexia y el dolor crónico. Los pacientes consumían una media de $9(3,7)$ fármacos y habían ingresado en un hospital $2,2(1,5)$ veces en el último año.

\section{Supervivencia}

Durante el seguimiento 86 (40,4\%) pacientes fallecieron en los primeros 6 meses y 107 (50,2\%) en el primer año. Los pacientes fallecidos eran de mayor edad, con más frecuencia vivían en residencia y tenían menor capacidad para las actividades básicas de la
Tabla 1

Características de los pacientes vivos y fallecidos durante el seguimiento de 12 meses

\begin{tabular}{|c|c|c|c|c|}
\hline & $\begin{array}{l}\text { Total } \\
\mathrm{N}=213\end{array}$ & $\begin{array}{l}\text { Vivos } \\
n=106\end{array}$ & $\begin{array}{l}\text { Fallecidos } \\
\mathrm{n}=107\end{array}$ & $\mathrm{p}$ \\
\hline Edad $^{\mathrm{a}}$ & $83(7)$ & $81,6(7,1)$ & $84,4(6,6)$ & 0,003 \\
\hline Sexo femenino & $106(49,8)$ & $54(50,9)$ & $52(48,6)$ & 0,732 \\
\hline Viven en residencia & $74(34,7)$ & $25(23,6)$ & $49(45,8)$ & 0,001 \\
\hline \multicolumn{5}{|l|}{ ECNOFA } \\
\hline Neurológica & $109(51,2)$ & $42(39,6)$ & $67(62,6)$ & 0,001 \\
\hline Respiratoria & $55(25,8)$ & $29(27,4)$ & $26(24,3)$ & 0,610 \\
\hline Cardíaca & $51(23,9)$ & $31(29,2)$ & $20(18,7)$ & 0,071 \\
\hline Renal & $38(17,8)$ & $22(20,8)$ & $16(15,0)$ & 0,269 \\
\hline Hepática & $9(4,2)$ & $5(4,7)$ & $4(3,7)$ & 0,723 \\
\hline Dos o más ECNOFA & $49(23,0)$ & $23(21,7)$ & $26(24,3)$ & 0,652 \\
\hline \multicolumn{5}{|l|}{ Categorías de PP } \\
\hline A. Cardíacas & $132(62)$ & $68(64,2)$ & $64(59,8)$ & 0,514 \\
\hline B. Riñón y vasculitis & $98(46)$ & $52(49,1)$ & $46(43,0)$ & 0,374 \\
\hline C. Respiratoria & $87(40,8)$ & $44(41,5)$ & $43(40,2)$ & 0,844 \\
\hline D. Digestiva & $12(5,6)$ & $6(5,7)$ & $6(5,6)$ & 0,987 \\
\hline E. Neurológica & $126(59,2)$ & $51(48,1)$ & $75(70,1)$ & 0,001 \\
\hline F. Diabetes y arteriopatía & $35(16,4)$ & $19(17,9)$ & $16(15,0)$ & 0,558 \\
\hline G. Anemia y neoplasia & $36(16,9)$ & $17(16)$ & $19(17,8)$ & 0,738 \\
\hline H. Osteomuscular & $33(15,5)$ & $16(15,1)$ & $17(15,9)$ & 0,873 \\
\hline Índice de Charlson ${ }^{\mathrm{a}}$ & $3,3(1,6)$ & $3,4(1,7)$ & $3,2(1,5)$ & 0,364 \\
\hline Índice de Barthel ${ }^{\mathrm{a}}$ & $42(36)$ & $55(36)$ & $28(31)$ & $<0,001$ \\
\hline$S P M S Q^{a}$ & $5,1(3,9)$ & $3,8(3,6)$ & $6,5(3,7)$ & $<0,001$ \\
\hline Enfermedad terminal & $90(42,3)$ & $30(28,3)$ & $60(56,1)$ & $<0,001$ \\
\hline \multicolumn{5}{|l|}{ Síntomas de enfermedad terminal } \\
\hline Insomnio & $51(23,9)$ & $20(18,9)$ & $31(29)$ & 0,084 \\
\hline Dolor crónico & $56(26,3)$ & $31(29,2)$ & $25(23,4)$ & 0,330 \\
\hline Úlceras por presión & $34(16)$ & $7(6,6)$ & $27(25,7)$ & $<0,001$ \\
\hline Anorexia & $59(27,7)$ & $23(21,7)$ & $36(33,6)$ & 0,051 \\
\hline Astenia & $29(13,6)$ & $15(14,2)$ & $14(13,1)$ & 0,820 \\
\hline Náuseas/vómitos & $14(6,6)$ & $8(7,5)$ & $6(5,6)$ & 0,568 \\
\hline Diarrea & $5(2,3)$ & $3(2,8)$ & $2(1,9)$ & 0,643 \\
\hline Estreñimiento & $68(31,9)$ & $34(32,1)$ & $34(31,8)$ & 0,963 \\
\hline Edemas & $70(32,9)$ & $36(34)$ & $34(31,8)$ & 0,734 \\
\hline Disnea de reposo & $44(20,7)$ & $21(19,8)$ & $23(21,5)$ & 0,761 \\
\hline Delirium & $38(17,8)$ & $15(14,2)$ & $23(21,5)$ & 0,162 \\
\hline Ingresos en últimos 12 meses $^{\mathrm{a}}$ & $2,2(1,5)$ & $2,2(1,5)$ & $2,3(1,5)$ & 0,556 \\
\hline Ingresos en últimos 3 meses $^{\mathrm{a}}$ & $1,4(0,7)$ & $1,4(0,6)$ & $1,5(0,7)$ & 0,283 \\
\hline Albúmina $(\mathrm{g} / \mathrm{dl})^{\mathrm{a}}$ & $3,1(0,6)$ & $3,2(0,6)$ & $2,9(0,6)$ & $<0,001$ \\
\hline Hemoglobina $(\mathrm{g} / \mathrm{dl})^{\mathrm{a}}$ & $11,5(1,9)$ & $11,7(1,8)$ & $11,2(2)$ & 0,039 \\
\hline Número de fármacos ${ }^{\mathrm{a}}$ & $9,0(3,7)$ & $9,7(3,9)$ & $8,4(3,5)$ & 0,013 \\
\hline Índice PROFUND & $9,8(4,2)$ & $8,5(3,9)$ & $11,2(4,2)$ & $<0,001$ \\
\hline Índice PALIAR & $5,2(4,2)$ & $3,6(3,1)$ & $6,7(4,6)$ & $<0,001$ \\
\hline
\end{tabular}

ECNOA: enfermedad crónica no oncológica en fase avanzada; PP: pluripatología; SPMSQ: Short Portable Mental Status Questionnaire.

Los datos se expresan en valor absoluto (porcentaje) y

a media (desviación estándar).

vida diaria y más deterioro cognitivo (tabla 1). Las enfermedades neurológicas fueron más frecuentes en estos pacientes. Los pacientes fallecidos padecían más úlceras por presión, consumían menos fármacos y tenían niveles más bajos de albúmina y hemoglobina. También tenían puntuaciones más altas en los índices PALIAR [6,7 $(4,6)$ frente a 3,6 (3,1); $\mathrm{p}<0,001]$ y PROFUND [11,2 (4,2) frente a 8,5 $(3,9) ; \mathrm{p}<0,001]$.

Al estratificar a los pacientes según los niveles de riesgo del índice PALIAR, 42 (19,7\%) pacientes tenían un riesgo bajo de muerte a los 6 meses, 66 (31\%) un riesgo intermedio, 59 (27,7\%) un riesgo alto y $46(21,6 \%)$ un riesgo muy alto. Conforme aumentaba el riesgo disminuyó la supervivencia (fig. 1A). Cuando se utilizó el índice PROFUND, 7 (3,3\%) pacientes tenían riesgo bajo, 47 (22,1) riesgo intermedio, 66(31\%) riesgo alto y $93(43,7 \%)$ riesgo muy alto. Cuanto mayor el riesgo menor fue la supervivencia (fig. 1B).

\section{Comparación de los índices PALIAR y PROFUND}

La correlación entre ambos índices fue intermedia (correlación de Pearson 0,436; p<0,001) (fig. 2). 

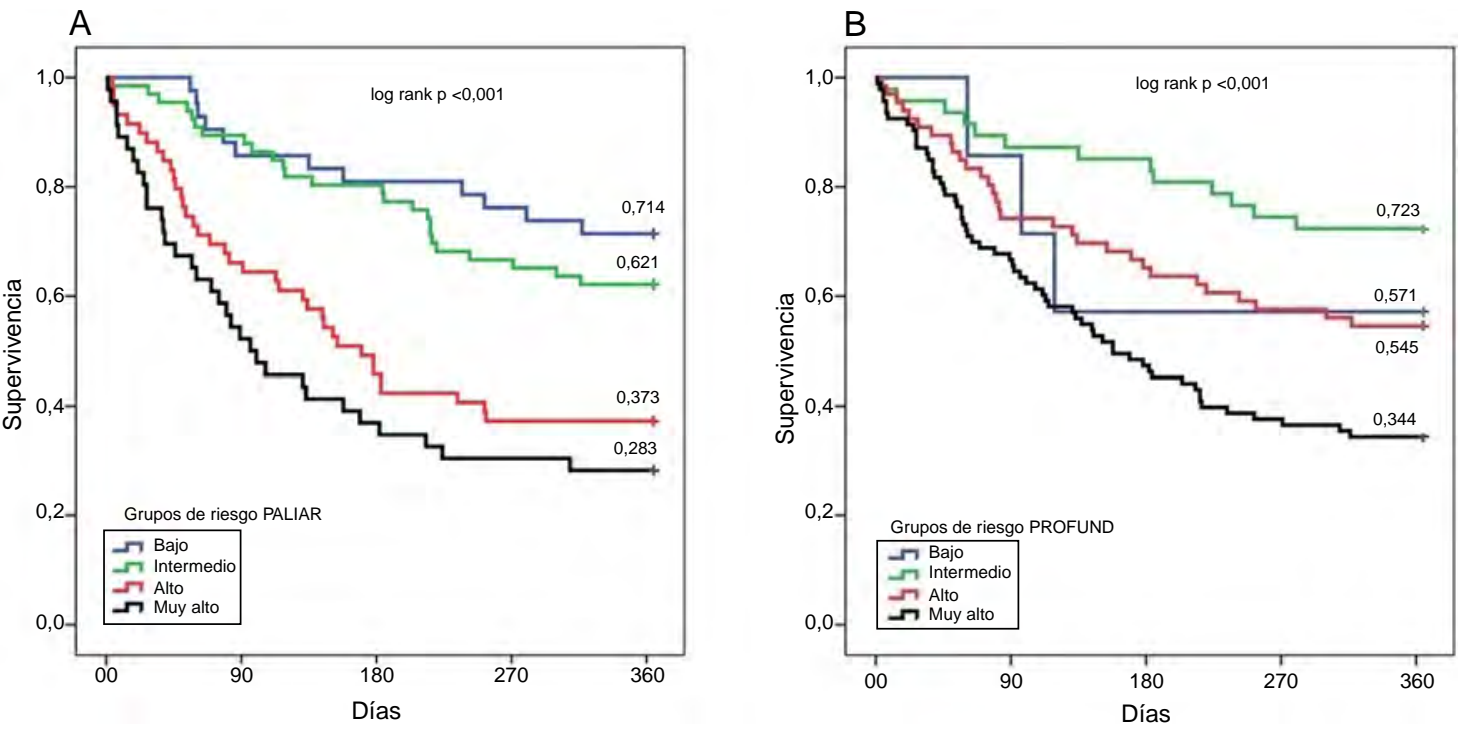

Figura 1. Curvas de supervivencia de Kaplan-Meier según los grupos de riesgo del índice PALIAR (1A) y del índice PROFUND (1B).

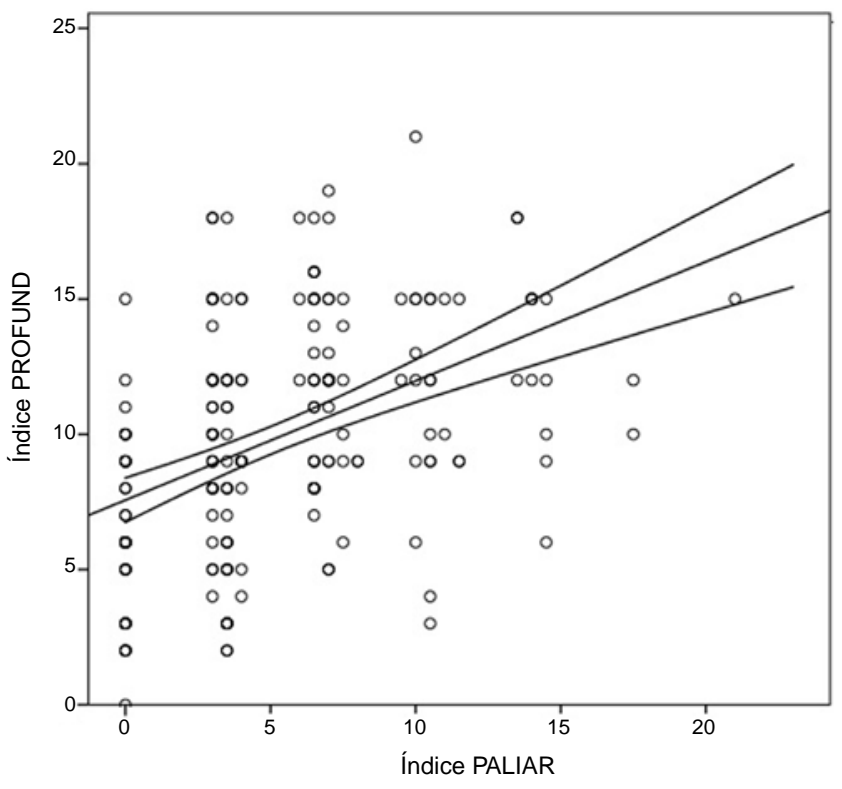

Figura 2. Correlación entre los índices PROFUND y PALIAR.

En la figura 3 se presentan las curvas ROC de los índices PALIAR y PROFUND. El índice PALIAR tuvo una mejor rentabilidad para predecir la mortalidad a los 6 meses (área bajo la curva de 0,734; IC95\%: 0,665-0,803; $\mathrm{p}<0,001$ ) que el índice PROFUND (0,677; IC95\%: 0,603-0,751), aunque a los 12 meses (área bajo la curva de 0,701; IC95\%: 0,632-0,771; $<<0,001)$ fue prácticamente similar a la del índice PROFUND (0,683; IC95\%: 0,611-0,754).

\section{Discusión}

Los principales hallazgos de nuestro estudio fueron que los PP con ECNOFA son ancianos y tienen deterioro funcional y cognitivo. Su mortalidad a los 6 y 12 meses fue alta, y el índice PALIAR tuvo mejor rendimiento que el índice PROFUND para predecir la muerte a los 6 meses.

Nuestros pacientes presentan diferencias con los de otros estudios. Al compararlos con la cohorte del proyecto PROFUND son de mayor edad, con más prevalencia de enfermedades neurológicas y renales y menor de enfermedades cardíacas y respiratorias. Además, tienen mayor discapacidad y más deterioro cognitivo ${ }^{2}$. Cuando se comparan con los enfermos del proyecto PALIAR se observan las mismas diferencias ${ }^{3}$. La estructura de la población aragonesa, que tiene una edad media 1,6 años mayor a la española puede explicar estas diferencias ${ }^{12}$. Las enfermedades del sistema nervioso aumentan con la edad y provocan una mayor discapacidad. Sin embargo, si los comparamos con un estudio con PP realizado en Aragón los pacientes de este estudio son de mayor edad, con más discapacidad funcional y cognitiva ${ }^{4}$. Esto indica que conforme avanza la edad de los PP es más probable que presenten ECNOFA y que estas se acompañen de mayor dependencia.

La mitad de los pacientes fallecieron en un año, pero hasta el $80 \%$ de la mortalidad se concentró en los primeros 6 meses. En el estudio PALIAR, que incluyó a pacientes con ECNOFA, la mortalidad a los 6 meses fue del 37,7\% cifra muy similar a la observada por nosotros. Sin embargo la mortalidad al año fue del 38-39\% en los estudios PROFUND y PLUPAR con $\mathrm{PP}^{2,4}$. Estos datos, además de confirmar la gravedad de estos pacientes, indican que cuando un PP desarrolla una ECNOFA la evolución de la enfermedad se acelera con la consiguiente disminución en el tiempo de supervivencia.

Existen índices pronósticos para las enfermedades más prevalentes, como por ejemplo los índices BODE y CODEX para pacientes con enfermedad pulmonar obstructiva crónica ${ }^{13,14}$, o el Seattle Heart Failure Model para pacientes con insuficiencia cardíaca ${ }^{15}$. Sin embargo, el envejecimiento y la multimorbilidad se han convertido en un reto para los sistemas sanitarios tal y como lo recoge el informe de la $\mathrm{OCDE}^{16}$. Según el mismo solo el 3\% de los pacientes con insuficiencia cardíaca, el $5 \%$ con demencia o el $18 \%$ con EPOC tienen la enfermedad de forma aislada. En nuestro estudio todos los pacientes son PP y hasta uno de cada 4 tiene más de una ECNOFA. Como ya se sabe la pluripatología es muy frecuente en los servicios de Medicina Interna y Geriatría ${ }^{17}$. Por ello en estos pacientes es más apropiado utilizar índices específicamente desarrollados para ellos, como los índices PALIAR y PROFUND. El primero establece el pronóstico a los 6 meses y el segundo al cabo de un año. Ambos son índices multidimensionales lo cual les da fortaleza en sus predicciones. Hasta nuestro conocimiento, no hay estudios que hayan comparado ambos índices cuando las ECNOFA aparecen en PP. En nuestro estudio el índice PALIAR fue ligeramente superior a los 6 meses y similar al índice PROFUND a los 12 meses. Por ello proponemos que cuando los PP desarrollan ECNOFA o en los pacientes con ECNOFA se cumplen criterios de PP se aplique 
A

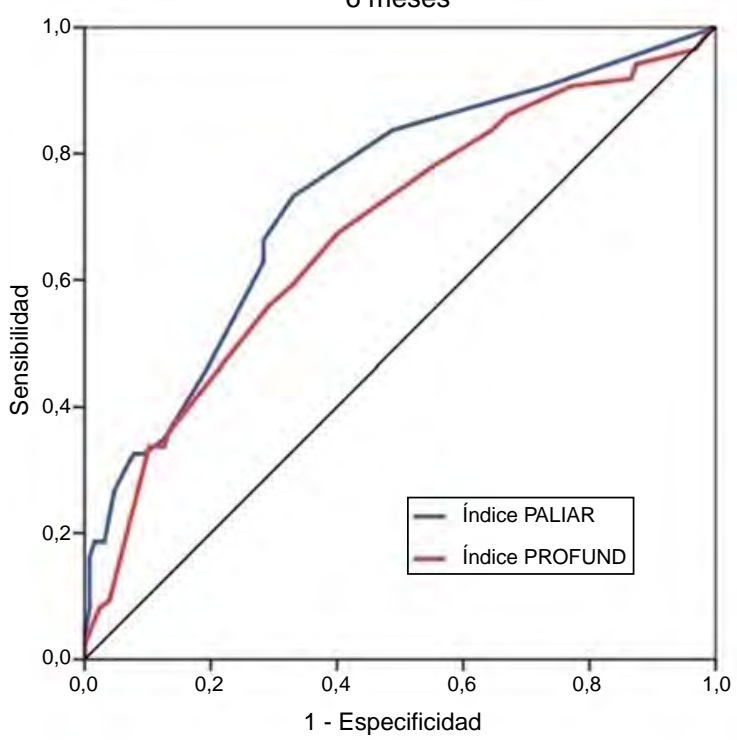

B

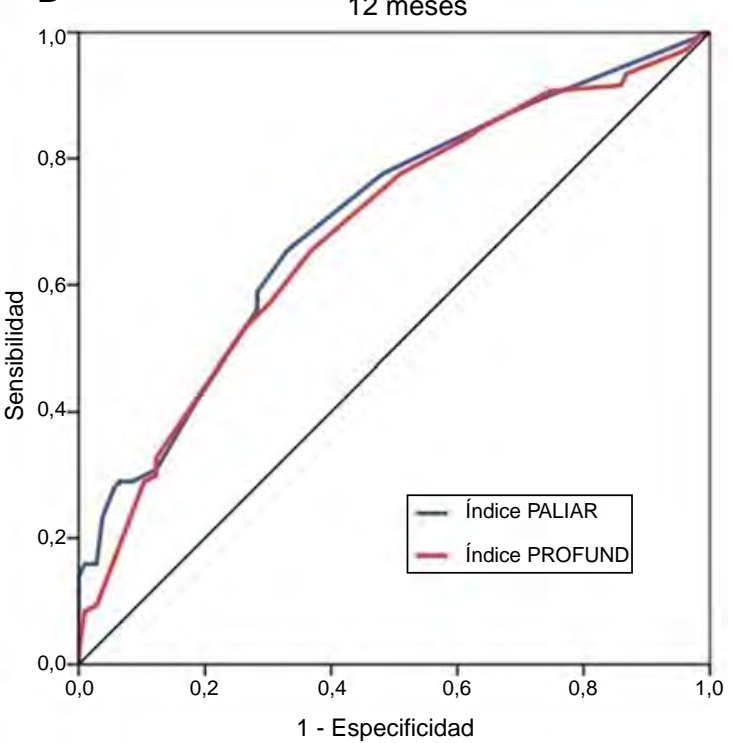

Figura 3. Curvas ROC de los índices PROFUND y PALIAR a los 6 y 12 meses.

preferentemente el índice PALIAR para establecer la probabilidad de muerte.

Nuestro estudio tiene algunas fortalezas. Fue un estudio multicéntrico, sin pérdidas en el seguimiento, con participación de hospitales comarcales y hospitales regionales de referencia, y con reclutamiento de pacientes procedentes tanto de zonas urbanas como rurales. Estos aspectos refuerzan sus resultados. Sin embargo, el estudio también tiene varias limitaciones. En primer lugar, solo se incluyeron pacientes hospitalizados y se excluyeron los pacientes fallecidos durante el ingreso. Esto puede suponer un sesgo de selección al haber excluido a pacientes con mayor mortalidad y podría limitar la aplicación de sus resultados a pacientes no ingresados. En segundo lugar, el estudio se ha realizado en la subcohorte de pacientes con PP incluidos en el estudio PALIARAGON que incluyó a pacientes con ECNOFA. Este análisis estaba previsto y predefinido en el protocolo del estudio. Sin embargo, aunque no parece probable, no podemos descartar que pudiera haber pequeños cambios en los resultados si el proceso hubiera sido el inverso, PP que desarrollan ECNOFA.

En resumen, con el envejecimiento progresivo de la población y los avances en cuidados de salud es frecuente la coexistencia de PP y ECNOFA. En estos casos proporcionar a cada paciente los recursos sanitarios que más se ajustan a sus expectativas de vida exige conocer su probabilidad de supervivencia, lo cual ayuda a planificar los cuidados de salud y la gestión de casos. La utilización del índice PALIAR podría ayudar a tomar estas decisiones.

\section{Financiación}

Cofinanciado con los Fondos Europeos de Desarrollo Regional (FEDER)

\section{Conflicto de intereses}

Los autores declaran no tener conflictos de intereses.

\section{Anexo. Material adicional}

Se puede consultar material adicional a este artículo en su versión electrónica disponible en https://doi.org/ 10.1016/j.medcli.2019.01.034.

\section{Anexo 1. Lista de investigadores del estudio PALIARAGON}

Jesús Díez-Manglano, Carolina Clemente-Sarasa, Daniel FuertesRuiz, Esperanza Bejarano-Tello, Eulalia Munilla López, María Pilar Lambán Aranda (Servicio de Medicina Interna, Hospital Royo Villanova, Zaragoza), Noelia Gómez Aguirre (Servicio de Medicina Interna, Hospital Ernest Lluch, Calatayud), José Velilla Marco, Carmen Bueno Castel, Alfonso García Aranda, Esther Artajona Rodrigo (Servicio de Medicina Interna, Hospital Universitario Miguel Servet, Zaragoza), Begoña de Escalante Yangüela, Borja Gracia Tello (Servicio de Medicina Interna, Hospital Clínico Universitario Lozano Blesa, Zaragoza), José Luis Cabrerizo García, Sandra Yagüe Vallejo (Servicio de Medicina Interna, Hospital General de la Defensa, Zaragoza).

\section{Bibliografía}

1. Ollero-Baturone M, Alvarez M, Barón-Franco B, Bernabéu-Wittel M, Codina A, Fernández-Moyano A, et al. Atención al paciente pluripatológico. Proceso asistencial integrado. 2. ${ }^{\mathrm{a}}$ ed Sevilla: Consejería de Salud. Junta de Andalucía; 2007. Disponible en: http://www.juntadeandalucia.es/servicios/publicaciones/detalle/50328.html [útimo acceso 26 Jul 2018].

2. Bernabeu-Wittel M, Ollero-Baturone M, Moreno-Gaviño L, Barón-Franco B, Fuertes A, Murcia-Zaragoza J, et al. Development of a new predictive model for polypathological patients. The PROFUND index. Eur J Intern Med. 2011;22:311-7.

3. Bernabeu-Witell M, Murcia Zaragoza J, Hernández Quiles C, Escolano Fernández B, Jarava Rol G, Oliver M, et al. Development of a six-month prognostic index in patients with advanced chronic medical conditions: The PALIAR Score. J Pain Symptom Manage. 2014:47:551-65.

4. Díez-Manglano J, Cabrerizo García JL, García-Arilla Calvo E, Jimeno Saínz A, Calvo Beguería E, Martínez-Álvarez RM, et al. External validation of the PROFUND index in polypathological patients from internal medicine and acute geriatrics departments in Aragón. Intern Emerg Med. 2015;10: 915-26.

5. Gómez-Aguirre N, Fuertes-Ruiz D, Gracia-Tello B, Clemente-Sarasa C, ArtajonaRodrigo E, Cabrerizo-García JL, et al. External validation of the PALIAR index for patients with advanced, nononcologic chronic diseases. Aging Clin Exp Res. 2018, http://dx.doi.org/10.1007/s40520-018-0980-3.

6. Charlson ME, Pompei P, Ales KL, MacKenzie CR. A new method of classifying prognostic comorbidity in longitudinal studies: Development and validation. J Chronic Dis. 1987;40:373-83.

7. Mahoney FI, Barthel DW. Functional evaluation: The Barthel index. Md State Med J. 1965:4:61-5.

8. Pfeiffer E. A short portable mental status questionnaire for the assessment of organic brain deficit in elderly patients. J Am Geriatr Soc. 1975;23:433-41. 
9. Oken MM, Creech RH, Tormey DC, Horton J, Davis TE, McFadden ET, et al. Toxicity and response criteria of the Eastern Cooperative Oncology Group. Am J Clin Oncol. 1982;5:649-55.

10. National Hospice Organization (NHO). Medical guidelines for determining prognosis in selected noncancer diseases. Hospice J. 1996;11:47-59.

11. Îndice Nacional de Defunciones [último acceso 24 Jul 2018]. Disponible en: https://www.msssi.gob.es/estadEstudios/estadisticas/estadisticas/estMinisterio/ IND_TipoDifusion.htm.

12. Instituto Aragonés de Estadística. Indicadores de estructura demográfica [último acceso 26 Jul 2018]. Disponible en: http://www.aragon.es/DepartamentosOrganismosPublicos/Institutos/Instituto AragonesEstadistica/AreasTematicas/02_Demografia_Y_Poblacion/02 Indicadores_demograficos/ci.01_Indicadores_estructura.detalleDepartamento? channelSelected=0\#section 1 .

13. Celli BR, Cote CG, Marin JM, Casanova C, Montes de Oca M, Mendez RA, et al. The body-mass index, airflow obstruction, dyspnea and exercise capacity index in chronic obstructive pulmonary disease. N Engl J Med. 2004;350:1005-12.
14. Almagro P, Soriano J, Cabrera FJ, Boixeda R, Alonso-Ortiz MB, Barreiro B, et al., and the working group on COPD Spanish Society of Internal Medicine. Shortand medium-term prognosis in patients hospitalized for COPD exacerbation. The CODEX index. Chest. 2014:145:972-80.

15. Levy WC, Mozaffarian D, Linker DT, Sutradhar SC, Anker SD, Cropp AB, et al. The Seattle Heart Failure Model Prediction of survival in heart failure. Circulation. 2006;113:1424-33.

16. OECD. Health reform: Meeting the challenge of ageing and multiple morbidities, OECD Publishing (2011) [último acceso 30 Jul 2018]. Disponible en: https://www.oecd-ilibrary.org/social-issues-migration-health/healthreform 9789264122314-en.

17. Díez-Manglano J, de Escalante Yangüela B, García-Arilla Calvo E, Ubis Díez E, Munilla López E, Clerencia sierra M, et al., on behalf of the PLUPAR study researchers. Differential characteristics in polypathological inpatients in internal medicine departments and acute geriatric units: The PLUPAR study. Eur J Intern Med. 2013;24:767-71. 